\title{
Tip-Enhanced Raman Nano-Spectroscopy of Smooth Spherical Gold Nanoparticles
}

Ashish Bhattarai, ${ }^{1}$ Zhihua Cheng, ${ }^{2}$ Alan G. Joly, ${ }^{1}$ Irina V. Novikova, ${ }^{3}$ James E. Evans, ${ }^{3}$ Zachary D. Schultz, ${ }^{4}$ Matthew R. Jones, ${ }^{2}$ and Patrick Z. El-Khoury ${ }^{1, *}$

${ }^{1}$ Physical Sciences Division, Pacific Northwest National Laboratory, P.O. Box 999, Richland, WA 99352, USA $;{ }^{2}$ Department of Chemistry, Department of Materials Science \&

Nanoengineering, Rice University, 6100 Main Street, Houston, TX 77005; ${ }^{3}$ Environmental and Molecular Sciences Laboratory, Pacific Northwest National Laboratory, P.O. Box 999, Richland, WA 99352, USA; ${ }^{4}$ Department of Chemistry and Biochemistry, The Ohio State University, Columbus, OH 43210, USA *patrick.elkhoury@pnnl.gov 


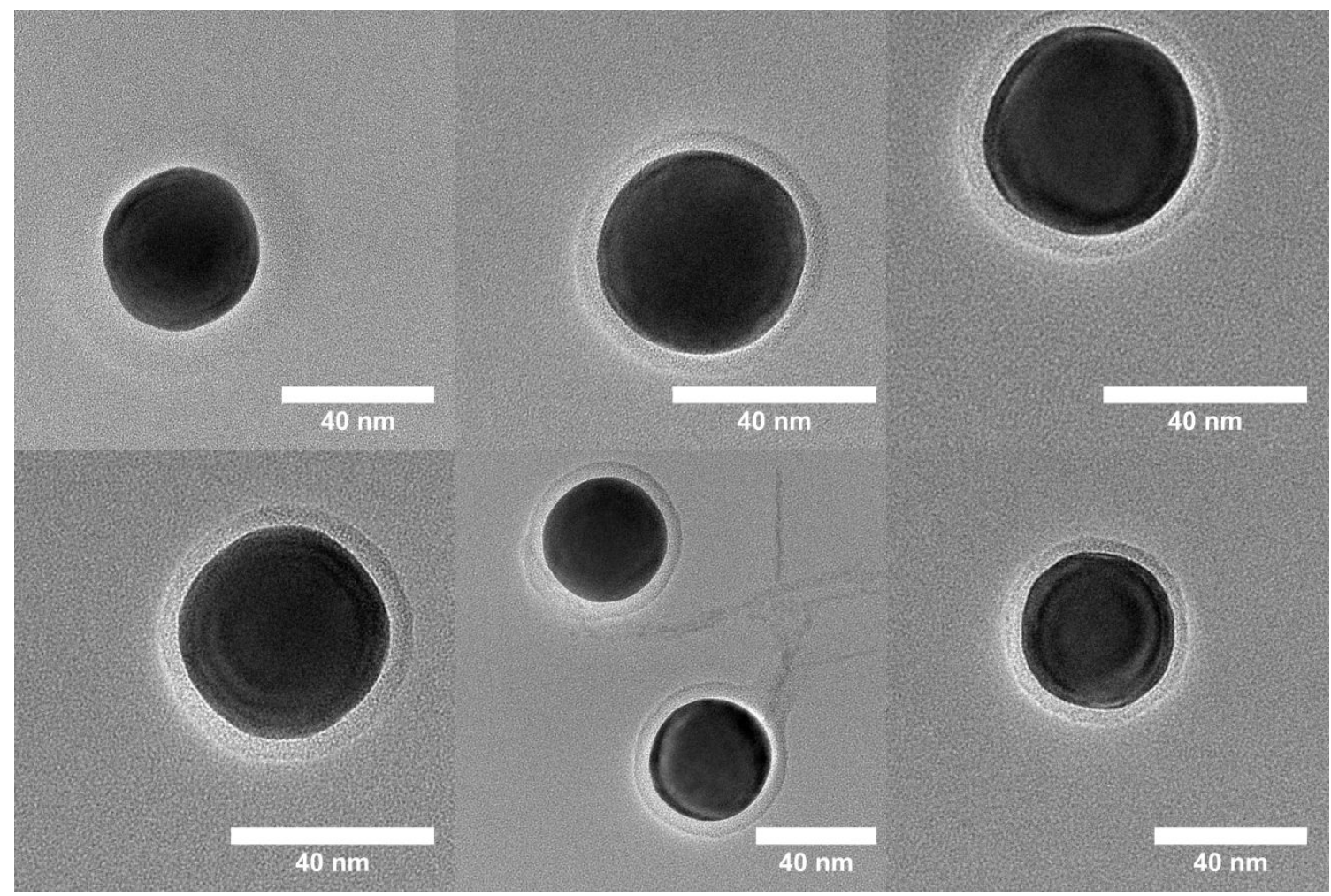

Figure S1. Representative transmission electron microscopy images of the Au nanospheres used in this study. A uniform thin layer of capping agents is visible in the image. 

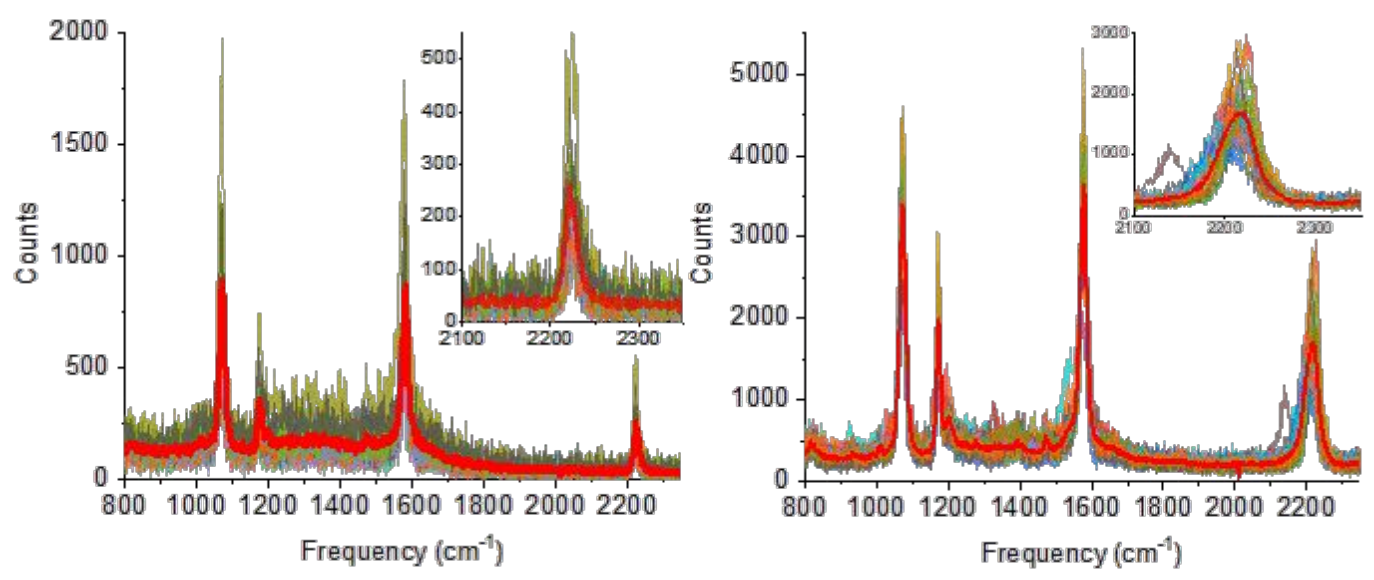

Figure S2. Collection of TERS spectra taken towards the center (left panel) and along the horseshoe scattering profile (right panel). These spectra form the basis of the analysis represented in Figure 4 of the main text. 

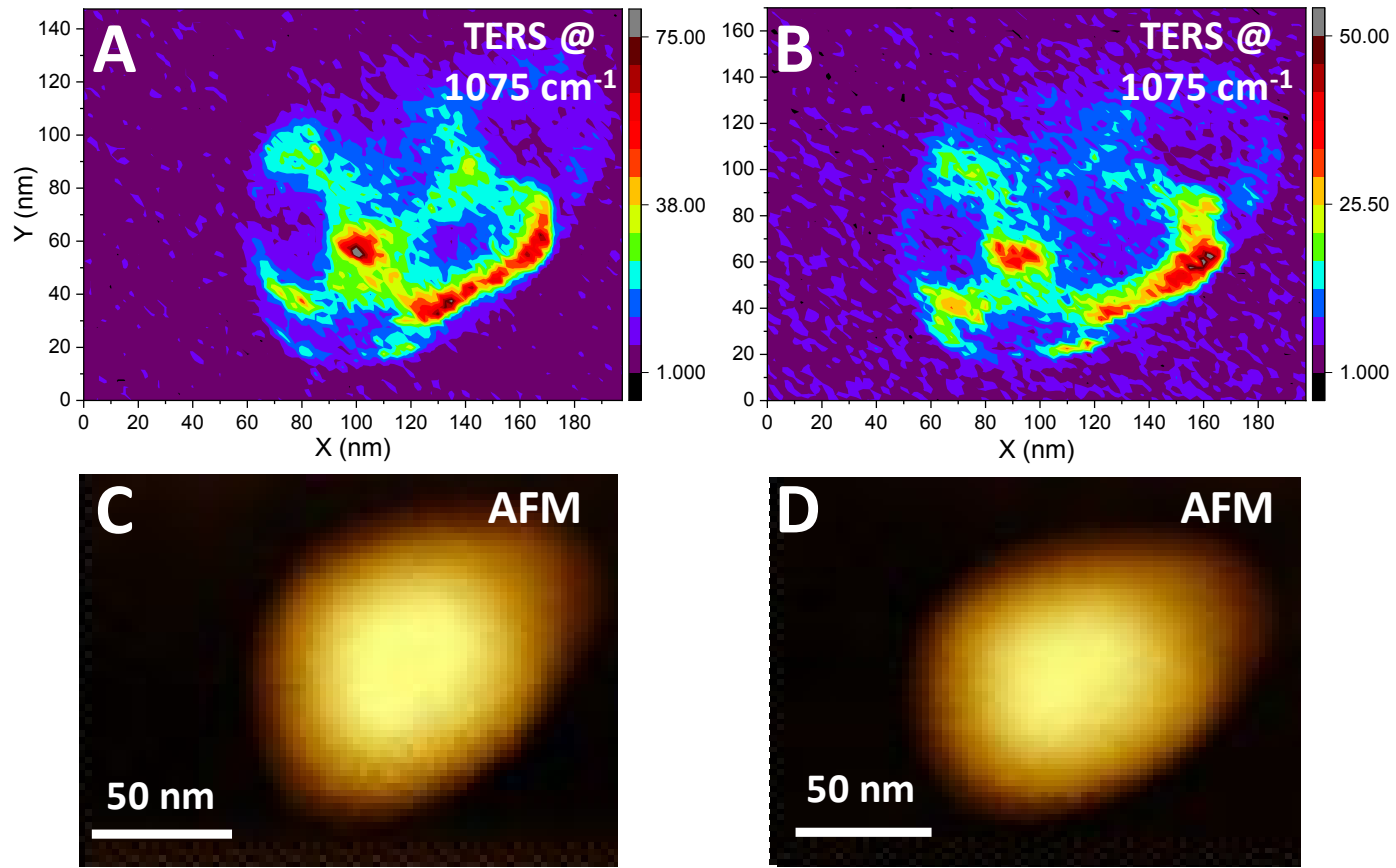

Figure S3. Artifacts encountered throughout the course of TERS mapping of spherical gold nanoparticles with a compromised probe are shown. The chemical (A-B) and topographic (C-D) images shown were recorded using the same probe (distinct from the ones used to record the images shown in the main text). Two different spheres are shown (A,C vs B,D). Both drifting and tip (self)imaging are evident in the recorded TERS and AFM maps. 

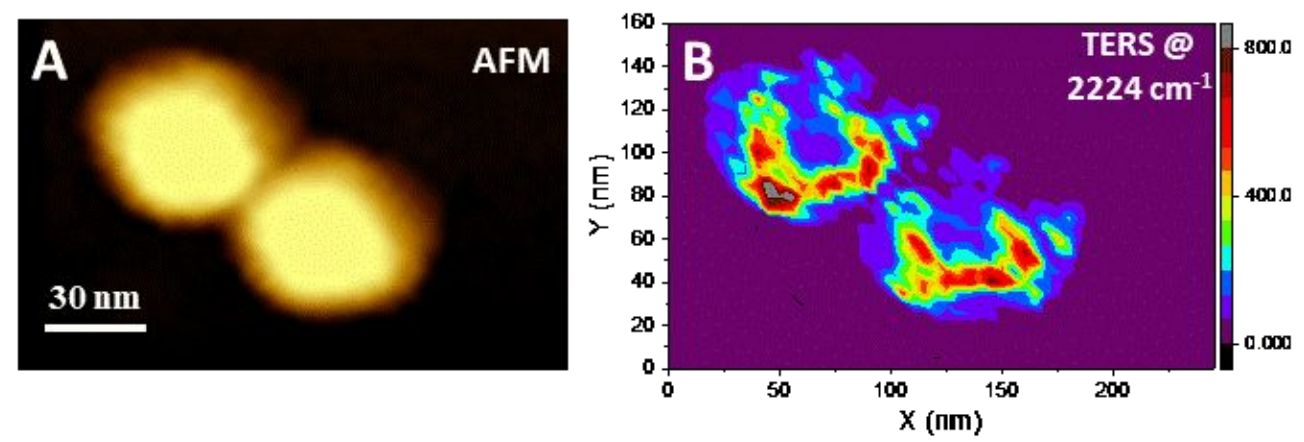

Figure S4. Simultaneously recorded AFM (A) and TERS (B) images of a nanosphere dimer. Shown is the same nanostructure analyzed in Figure 3 of the main text, but herein following spolarized light irradiation. 

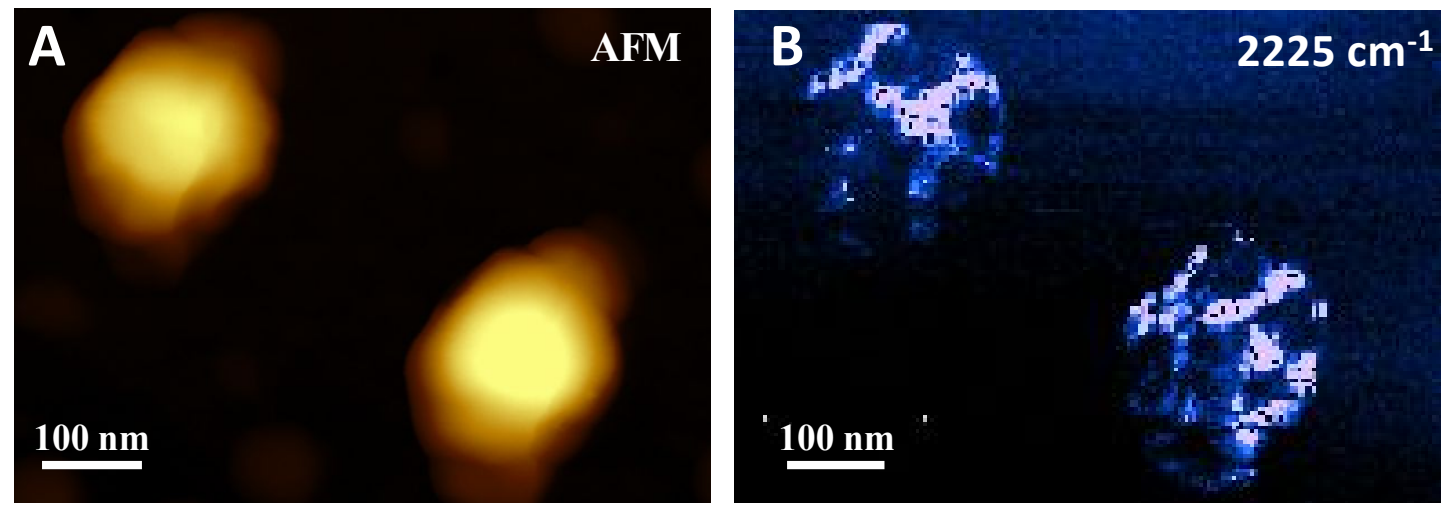

Figure S5. Simultaneously recorded AFM (A) and TERS (B) images of faceted silver nanoparticles. See reference 23 of the main text for more details. 